\title{
APLICACIÓN DE LA PROPUESTA GRUPOS DE INTERAPRENDIZAJE -GIA- EN EL DESEMPEÑO DOCENTE EN UNA INSTITUCIÓN EDUCATIVA DE TACNA.
}

\author{
APPLICATION OF THE INTERLEARNING GROUPS PROPOSAL -GIA- IN THE TEACHING PERFORMANCE \\ IN AN EDUCATIONAL INSTITUTION IN TACNA
}

\author{
María del Carmen Ayquipa Granda ${ }^{1}$ \\ http://orcid.org/0000-0003-2038-434X. \\ mariadelcarmen-0326@hotmail.com \\ Marcelino Raúl Valdivia Dueñas ${ }^{2}$ \\ http://orcid.org/0000-0002-9985-1651. \\ marcelinovd@gmail.com
}

Aceptado: 17/02/2021 Publicado online:30/05/2021

\begin{abstract}
RESUMEN
Investigación básica, explicativa. Busca determinar el impacto de la aplicación de la estrategia "Grupos de Interaprendizaje" (GIA) en el desempeño de los profesores de una institución educativa en Tacna. Se trabajó con una población de 62 unidades de estudio: 27 docentes del nivel primaria y 35 docentes del nivel secundario. La recolección de la información se realizó a través de dos cuestionarios: uno para recoger información sobre la aplicación de la estrategia GIA, y otro, sobre la información del nivel del desempeño docente. Se ha determinado que el grado de aplicación de la estrategia GIA en la Institución Educativa ha sido óptimo en $51.61 \%$. Sin embargo, se encuentran por debajo de este porcentaje indicadores como: distribución del tiempo empleado (32.26\%), organización (46.77\%), recursos empleados $33.87 \%$ y nivel de control (40.32\%). Para establecer la relación entre las variables se aplicó la prueba de Rho de Spearman, el coeficiente obtenido fue 0,120 y un $p$ valor igual a 0,353 ( $p$-valor $>0.05$ ) que representa una correlación prácticamente nula entre las variables de estudio. Finalmente, los resultados encontrados permiten concluir que la aplicación de la estrategia "Grupos de Interaprendizaje" (GIA) tuvo un impacto moderado en el desempeño docente.
\end{abstract}

Grupos de Interaprendizaje (GIA), desempeño docente, organización, ejecución, administración, interrelación, planificación.

\begin{abstract}
Basic, explanatory research. It seeks to determine the impact of the application of the strategy "Interlearning Groups" (GIA) on the performance of teachers in an educational institution in Tacna. We worked with a population of 62 study units: 27 primary school teachers and 35 secondary school teachers. Information was collected through two questionnaires: one to collect information on the application of the GIA strategy, and the other to collect information on the level of teacher performance. It has been determined that the degree of application of the GIA strategy in the educational institution has been optimal at $51.61 \%$. However, indicators such as: distribution of time spent (32.26\%), organization (46.77\%), resources used
\end{abstract}

\footnotetext{
1 Universidad Privada de Tacna. Magíster en Docencia Universitaria y Gestión Educativa.

2 Universidad Privada de Tacna. Facultad de Educación Ciencias de la Comunicación y Humanidades. Doctor en Educación
} 
(33.87\%) and level of control (40.32\%) are below this percentage. To establish the relationship between the variables, Spearman's Rho test was applied, the coefficient obtained was 0.120 and a $p$-value equal to 0.353 ( $p$-value $>0.05$ ), which represents a practically null correlation between the variables under study. Finally, the results found allow us to conclude that the application of the strategy "Interlearning Groups" (GIA) had a moderate impact on the teaching performance in the educational institution Manuel A. Odría in 2018.

Keywords: Interlearning Groups (GIA), teaching performance, organization, execution, administration, interrelationship, planning.

\section{INTRODUCCIÓN}

El desempeño docente se ha convertido en un factor básico que afecta la calidad de la educación. Los maestros planifican, ejecutan, interactúan, administran recursos y evalúan el aprendizaje. El progreso continuo de la educación requiere cambios importantes en el papel de los docentes en el proceso de enseñanza. Por ello, el estado se preocupa por la educación para mejorar el desempeño docente. En tal sentido, se han propuesto diversas alternativas para mejorar el desempeño de los docentes en las tareas educativas en diferentes etapas del proceso de enseñanza y aprendizaje. Uno de estos métodos alternativos es el Grupo de Interaprendizaje Docente GíA, una estrategia de formación para el acompañamiento que se ha desarrollado en diferentes instituciones educativas de Tacna. El propósito del estudio fue evaluar el impacto de la aplicación de la estrategia "Grupo Interactivo" (GÍA) en el desempeño docente de la Institución Educativa Manuel Odria en el 2018. En cuanto al docente, porque es él quien interactúa con los alumnos, gestiona los recursos didácticos y las evaluaciones para lograr el aprendizaje de los alumnos.

En el desarrollo del proceso de enseñanza, adoptamos las siguientes estrategias: activar conocimientos previos, orientar la atención de los estudiantes, promover la relación entre conocimientos previos y nuevos conocimientos, organizar la información de aprendizaje, y otras cuando el docente haya dominado el uso de estas estrategias, producirá un buen efecto de enseñanza. Sin embargo, basta no solo con realizar la labor educativa en el aula. Los profesores también deben dominar las estrategias de planificación, especialmente la correcta gestión de las evaluaciones. Estas estrategias son fundamentales para su desempeño en el aula y una mejor educación. Los programas de formación continua, de acuerdo a la Ley 30220 "buscan actualizar los conocimientos profesionales en aspectos teóricos y prácticos de una disciplina, o desarrollar y actualizar determinadas habilidades y competencias" (Congreso, 2014). De acuerdo a su finalidad, se podría colegir, lo que permitiría mejorar el desempeño del docente. El Ministerio de Educación, en estos últimos años, viene promoviendo diversas formas de capacitación obligatoria, para mejorar el desempeño docente. En la Institución Educativa "Manuel A Odría" en el año 2018 se llevó a cabo la experiencia GIA, en la que participaron profesores de primaria y Secundaria. De allí es que se considera que además de las evaluaciones oficiales, sería conveniente conocer el impacto que esta forma de trabajo tiene en el desempeño docente de los profesores que participaron en ella.

Mérida (2018), afirma que lo directores tienen conocimiento sobre la importancia de la evaluación del desempeño docente, sin embargo, este proceso no tiene una ejecución sistemática de la evaluación del desempeño, no utilizan un instrumento estructurado formalmente, y la periodificación de la misma no está definida. Así se revela la preocupación por el desempeño docente como un agente importante del sistema educativo para conseguir los logros esperados. Rodríguez (2015) observó que el desempeño docente es atribuible al 50,7\% de los grupos de acompañamiento docente y de aprendizaje cruzado en las instituciones educativas, de la información consultada se puede inferir que los docentes que participan en GIA se han visto afectados. La razón es que esto se refleja en su desempeño docente, que mejora la calidad de los servicios educativos que brinda la institución y beneficia indirectamente a los estudiantes. 
Chávez (2017), señala que el desempeño docente es regular lo que da lugar a que se investigue sobre esta variable, que requiere se busque alternativas para su mejora. Espinoza, Vilca y Pariona (2014) que la tasa efectiva de desempeño docente es del $48,6 \%$. Similar al desempeño docente con capacidad docente, responsabilidad profesional y relaciones interpersonales, este resultado refuerza la necesidad de evaluar las aplicaciones de GIA, las cuales están diseñadas para mejorar la efectividad de la enseñanza. La educación y la formación de los docentes que todavía es insuficiente por falta de inversión u otros factores son cruciales. Es en esta situación que la formación continua del profesorado es importante, y la alternativa propuesta como una de las posibles estrategias para ayudar a mejorar el desempeño docente se denomina Estrategia de Grupo de Interaprendizaje (GIA), que aborda este desafío Método factible.

Se considera que los GIA deben fortalecer el desempeño de los docentes, permitiendo a sus integrantes dialogar sobre la actividad docente, decidir y emprender acciones educativas, fomentando una actitud reflexiva, analítica y crítica desarrollando actitudes co-gestionarias y autogestionarias contribuyendo a la auto y mutua capacitación permanente y así lograr un crecimiento personal, despertando potencialidades, cambiando nuevas actitudes y conocimientos.

El Grupo de Interaprendizaje es el encuentro de un grupo de docentes para intercambiar experiencias pedagógicas, así como también estrategias didácticas, y de ese modo, optimizar, su desempeño MINEDU (2017).

En el año 2018, el MINEDU publica el Manual de aplicación "Rubricas de observación de aula para la Evaluación del Desempeño Docente". Para ello, considera cinco actuaciones que deben ser observadas: participación activa de los estudiantes en el proceso de aprendizaje; promoción del razonamiento, creatividad y/o pensamiento crítico; Evaluación del progreso del aprendizaje para retroalimentar a los estudiantes y adaptarse a su enseñanza, Promover un ambiente cercano y respetuoso y una regulación positiva del comportamiento de los estudiantes. Para la evaluación de los profesores en cinco actuaciones, se deben considerar cuatro niveles.

\begin{tabular}{llll} 
Nivel I & Nivel II & Nivel III & Nivel \\
& & & VI \\
\hline Muy deficiente & En Proceso & Suficiente & Destacado
\end{tabular}

\section{OBJETIVO DE LA INVESTIGACIÓN}

Determinar el impacto de la aplicación de la estrategia "Grupos de Interaprendizaje" (GIA) en el desempeño docente en la institución educativa Manuel A. Odría en el año 2018.

\section{METOdOLOGÍA}

Básica descriptiva. La investigación es micro regional. Unidades de estudio los docentes del nivel primaria y secundaria de la I.E. "Manuel A. Odría". La población estuvo conformada por 62 docentes de la I.E. "Manual A. Odría" del distrito de Ciudad Nueva correspondiendo 27 docentes al nivel primaria y 35 al nivel secundaria. Se trabajó con toda la población. Se aplicó la estrategia GIA: Planificación del GIA, tiempo, organización del GIA, recursos en el GIA, control de la ejecución del GIA y se evaluó el desempeño docente a través de los indicadores: Planificación de la actividad docente, Ejecución del proceso E-A, Interrelación docente-alumno, manejo de recursos para el aprendizaje, Administración de la evaluación del aprendizaje.

La recolección de la información se efectuó en forma personal y de primera fuente. Los instrumentos seleccionados para la recolección de datos fueron dos cuestionarios: El primero fue un cuestionario conformado por 15 preguntas o ítems distribuidas en los indicadores ya mencionados el cual se validó a través del Coeficiente Kuder-Richardson donde alcanzó un puntaje de 0.79 . Para la 
medición de la variable desempeño, se utilizó una escala de medición con las siguientes categorías y rangos: Aplicación óptima (21-29), aplicación regular (12-20) y aplicación pésima (02-11). El instrumento que se empleó fue un cuestionario conformado por 15 preguntas o ítems distribuidas en los indicadores ya mencionados, el cual se validó a través del Coeficiente Kuder-Richardson donde alcanzó un puntaje de 0.72 . Para la medición se utilizó una escala de medición con las siguientes categorías y rangos: Desempeño docente adecuado (26-31) y Desempeño docente no adecuado (1925).

\section{RESULTADOS}

\section{Estrategia "Grupos de Interaprendizaje" (GIA)}

La información sobre el grado de aplicación de la estrategia "Grupos de Interaprendizaje" se presenta en función a cinco indicadores que permitirán la evaluación final de la variable.

Tabla 1

Nivel de aplicación de los indicadores GIA en la I.E. Manuel A. Odría de Tacna 2018

\begin{tabular}{|c|c|c|c|c|c|c|}
\hline \multirow[b]{2}{*}{ Planificación } & \multicolumn{2}{|c|}{ Primaria } & \multicolumn{2}{|c|}{ Secundaria } & \multicolumn{2}{|c|}{ Total } \\
\hline & $f$ & $\%$ & $f$ & $\%$ & $f$ & $\%$ \\
\hline Bueno & 10 & 37.04 & 22 & 62.86 & 32 & 51.61 \\
\hline Regular & 12 & 44.44 & 13 & 37.14 & 25 & 40.32 \\
\hline Malo & 5 & 18.52 & 0 & 0.00 & 5 & 8.37 \\
\hline Total & 27 & 100.00 & 35 & 100.00 & 62 & 100.00 \\
\hline \multicolumn{7}{|l|}{ Tiempo } \\
\hline Bueno & 6 & 22.22 & 14 & 40.00 & 20 & 32.26 \\
\hline Regular & 11 & 40.74 & 16 & 45.71 & 27 & 43.55 \\
\hline Malo & 10 & 37.04 & 5 & 14.29 & 15 & 24.19 \\
\hline Total & 27 & 100.00 & 35 & 100.00 & 62 & 100.00 \\
\hline \multicolumn{7}{|l|}{ Organización } \\
\hline Bueno & 10 & 37.04 & 19 & 54.29 & 29 & 46.77 \\
\hline Regular & 9 & 33.33 & 10 & 28.57 & 19 & 30.65 \\
\hline Malo & 8 & 29.63 & 6 & 17.14 & 14 & 22.58 \\
\hline Total & 27 & 100.00 & 35 & 100.00 & 62 & 100.00 \\
\hline \multicolumn{7}{|l|}{ Recurso } \\
\hline Bueno & 8 & 29.63 & 13 & 37.14 & 21 & 33.87 \\
\hline Regular & 16 & 59.26 & 17 & 48.57 & 33 & 53.23 \\
\hline Malo & 3 & 11.11 & 5 & 14.29 & 8 & 12.90 \\
\hline Total & 27 & 100.00 & 35 & 100.00 & 62 & 100.00 \\
\hline \multicolumn{7}{|l|}{ Control } \\
\hline Bueno & 7 & 25.93 & 18 & 51.43 & 25 & 40.32 \\
\hline Regular & 13 & 48.15 & 14 & 40.00 & 27 & 43.55 \\
\hline Malo & 17 & 25.93 & 3 & 8.57 & 10 & 16.13 \\
\hline Total & 27 & 100.00 & 35 & 100.00 & 62 & 100.00 \\
\hline
\end{tabular}

Fuente: cuestionario aplicado a docentes

La tabla 1 presenta información sobre el Nivel aplicación de los GIA en la I.E. Manuel A. Odría de Tacna. 2018. Se aprecia que entre el nivel primaria y secundaria un $51.61 \%$ tuvo "Buena" aplicación de los indicadores GIA. De acuerdo a la información presentada se observa que las unidades de estudio de primaria manifiestan debilidades en la participación de los docentes para la planificación de los 
Grupos de Inter-Aprendizaje lo que determina que este indicador alcance el nivel regular en mayoría de porcentaje (44.44\%), mientras que en el nivel de secundaria el $62.86 \%$ manifiesta que la planificación del GIA se desarrolla de buena manera. Se observa que las unidades de estudio, entre el nivel primaria y secundaria, manifiestan que la planificación de los GIA cuenta en gran medida con un cronograma de actividades y un plan de trabajo elaborado de acuerdo a las necesidades, intereses, capacidades y dificultades que presentan los docentes de la institución, así como de la participación de docentes y directivos en su elaboración, que si bien determina un buen desarrollo de este indicador puede mejorarse a través de la toma de decisiones que mejoren la planificación de los GIA en beneficio de los estudiantes.

Sobre el Nivel de distribución del tiempo empleado en actividades en la aplicación de los GIA, se observa que entre el nivel primaria y secundaria un $43.35 \%$ se ubicó en la categoría "Regular", las unidades de estudio manifestaron que la distribución de las actividades de los GIA tuvo deficiencias puesto que no se incrementaron horas de trabajo debido a metas inconclusas del bimestre académico; sin embargo, expresaron que sí se consideró la disponibilidad de casi todos los docentes para su participación así como del trabajo conjunto entre docentes y directivos para elaborar el cronograma de trabajo de actividades por lo que entre el nivel de primaria y secundaria la distribución del tiempo es considerada regular.

Acerca del Nivel de organización en la aplicación de los GIA se aprecia que entre el nivel primaria y secundaria el $46.77 \%$ se ubicaron en la categoría "Buena", de acuerdo a la información presentada se interpreta que los docentes consideran que en general existió una buena organización del GIA, sin embargo los docentes del nivel primaria manifestaron que la motivación y sensibilización sobre el GIA se desarrolló con algunas debilidades, puesto que solo el $37.04 \%$ consideraron sentirse motivados y/o sensibilizados para su participación, de igual manera los docentes del nivel secundaria, a pesar de que consideraron en un 54.29\% la buena organización de los GIA, evidenciaron deficiencias en la distribución de funciones de acuerdo a las capacidades de los docentes. Por lo que la organización para la aplicación de los GIA puede mejorarse a través de un trabajo en equipo que refleje la identidad institucional, la motivación y sensibilización frente a esta situación.

El Nivel de los recursos empleados en actividades en la planificación de los GIA vemos que entre el nivel primaria y secundaria el $53.23 \%$ se ubicaron en la categoría "Regular", las unidades de estudio manifestaron que existieron dificultades para elaborar y compartir material didáctico para las sesiones de aprendizaje tanto en el nivel primaria y secundaria por lo que este indicador alcanzó un nivel regular con $59.26 \%$ y $48.57 \%$ respectivamente. Por otro lado, expresaron que, si hubo coordinación con el órgano directivo para la designación de los ambientes de trabajo y el mobiliario necesario ubicándose en general el porcentaje mayor entre ambos niveles educativos en la categoría "Regular", pudiendo realizarse mejoras para optimizar el desarrollo de este indicador.

Luego por el Nivel del control de las actividades en la aplicación de los GIA se observa que entre el nivel primaria y secundaria el $43.55 \%$ se ubicaron en la categoría "Regular", el control de las actividades, como indicador de medición de la aplicación del GIA, es el registro que se lleva antes, durante y después de las actividades programadas en cuanto a la participación de los docentes, su puntualidad, compromiso y participación. Los resultados obtenidos en el nivel primaria reflejan que los docentes evidenciaron dificultades para registrar sus experiencias pedagógicas lo cual influenció en los resultados del indicador, ubicándose la mayor parte del porcentaje en la categoría regular con un $48.15 \%$, caso contrario en el nivel secundaria que se evidenció un $51.43 \%$ de buen control de las actividades llevadas a cabo a través del registro de asistencia, puntualidad lo que refleja su compromiso y participación de los docentes en las jornadas de trabajo, es por ello que el nivel del control de actividades tanto entre el nivel primaria y secundaria se dio de forma regular.

La tabla 2 presenta información sobre el Grado de aplicación de la estrategia GIA en la Institución Educativa, se aprecia que entre el nivel primaria y secundaria el $51.61 \%$ se ubicó en la categoría "Aplicación óptima", los resultados obtenidos evidencian que la aplicación de los GIA se dio, en términos generales de forma óptima, sin embargo, el porcentaje alcanzado es el mínimo requerido para ubicarse en la categoría "Aplicación óptima" lo cual es el reflejo de que en el nivel primaria la 
aplicación de los GIA solo alcanzó, en gran porcentaje, la categoría “Aplicación regular" con un 48.15\% puesto que los docentes evidenciaron deficiencias significativas en el manejo de recursos y el control de las actividades programadas. En nivel secundario un $65.71 \%$ manifestaron que la aplicación fue óptima debido a que se realizaron acciones que permitieron una buena planificación, organización, y control de las actividades, sin embargo, existieron debilidades en la distribución del tiempo y el manejo de recursos lo que ocasionó que si bien la aplicación es óptima es a través de un bajo porcentaje.

Tabla 2

Grado de aplicación de la Estrategia "Grupos de Interaprendizaje" (GIA) en la Institución Educativa Manuela A. Odría en el año 2018.

\begin{tabular}{lcccccc}
\hline \multirow{2}{*}{ Categorías } & \multicolumn{2}{c}{ Primaria } & \multicolumn{2}{c}{ Secundaria } & \multicolumn{2}{c}{ Total } \\
\cline { 2 - 7 } & $\mathrm{f}$ & $\%$ & $\mathrm{f}$ & $\%$ & $\mathrm{f}$ & $\%$ \\
\hline Aplicación óptima & 9 & 33.33 & 23 & 65.71 & 32 & 51.61 \\
Aplicación regular & 13 & 48.15 & 12 & 34.29 & 25 & 40.32 \\
Aplicación pésima & 5 & 18.52 & 0 & 0.00 & 5 & 8.07 \\
Total & 27 & 100.00 & 35 & 100.00 & 62 & 100.00 \\
\hline
\end{tabular}

Fuente: cuestionario aplicado a docentes

\section{Desempeño docente}

La información sobre el nivel de Desempeño docente se presenta en función a cinco indicadores que permitirán la evaluación final de la variable.

Tabla 3

Desempeño docente según indicadores

\begin{tabular}{lcccccc}
\hline Planificación de la & \multicolumn{2}{c}{ Primaria } & \multicolumn{2}{c}{ Secundaria } & \multicolumn{2}{c}{ Total } \\
\cline { 2 - 7 } actividad docente & $\mathrm{f}$ & $\%$ & $\mathrm{f}$ & $\%$ & $\mathrm{f}$ & $\%$ \\
\hline Bueno & 11 & 40.74 & 15 & 42.86 & 26 & 41.94 \\
Regular & 16 & 59.26 & 18 & 51.43 & 34 & 54.84 \\
Malo & 0 & 0.00 & 2 & 5.71 & 2 & 3.22 \\
Total & 27 & 100.00 & 35 & 100.00 & 62 & 100.00 \\
\hline Ejecución del proceso E-A & & & & & \\
\hline Adecuada & 21 & 77.78 & 20 & 57.14 & 41 & 66.13 \\
Inadecuada & 6 & 7.14 & 15 & 42.86 & 21 & 33.87 \\
Total & 27 & 84.92 & 35 & 100.00 & 62 & 100.00 \\
\hline Interrelación docente-alumno & & & & & \\
\hline Bueno & 18 & 66.67 & 20 & 57.14 & 38 & 61.29 \\
Regular & 7 & 25.93 & 14 & 40.00 & 21 & 33.87 \\
Malo & 2 & 7.41 & 1 & 2.86 & 3 & 4.84 \\
Total & 27 & 100.00 & 35 & 100.00 & 62 & 100.00 \\
\hline Manejo de recurso & & & & & & \\
\hline Adecuada & 15 & 55.56 & 21 & 60.00 & 36 & 58.06 \\
Inadecuada & 12 & 44.44 & 14 & 40.00 & 26 & 41.94 \\
Total & 27 & 100.00 & 35 & 100.00 & 62 & 100.00 \\
\hline Administración de evaluación & & & & & \\
\hline Bueno & 10 & 37.04 & 6 & 17.14 & 16 & 25.81 \\
Regular & 14 & 51.85 & 25 & 71.43 & 39 & 62.90 \\
Malo & 3 & 11.11 & 4 & 11.43 & 7 & 11.29 \\
Total & 100.00 & 35 & 100.00 & 62 & 100.00 \\
\hline Fuente: cuestionario aplicado a docentes & & & & & \\
\hline
\end{tabular}


La tabla 3 presenta información sobre el desempeño docente, así el Nivel de la planificación que presentan los docentes en su desempeño, se observa que el $54.84 \%$ se ubicó en la categoría "Regular", en la categoría "Buena" se ubicaron 41.94\%. De acuerdo con la información presentada, la planificación que realizan los docentes es regular, puesto que en el nivel primaria un 59.26\% se ubicó en la categoría "Regular" ya que evidenciaron que la programación curricular se realizó, en mediana medida, de acuerdo a la realidad socioeconómica del aula lo mismo manifestaron los docentes del nivel secundaria además de deficiencias en la selección de contenidos en función de los aprendizajes que la escuela y la comunidad requieren obteniendo así la mayoría de porcentaje en la categoría "regular" con un $51.43 \%$.

Sobre el Nivel de ejecución que presentan los docentes se observa que, entre el nivel primaria y secundaria, en la categoría "Adecuada" se ubicaron el 66.13\%. La información presentada indica que los docentes ejecutan las actividades programadas adecuadamente, puesto que tanto el nivel primario como secundaria manifiestan que las actividades y/o estrategias que emplean despiertan el interés de los estudiantes y los orientan hacia una reflexión significativa sobre lo que aprende durante las sesiones de clase obteniendo un $77.78 \%$ y $57.14 \%$ respectivamente. Sin embargo, se evidenció que los docentes de la institución presentan algunas debilidades para contextualizar las sesiones de clase con los intereses, estilos e identidad cultural de los estudiantes.

En cuanto al Nivel de interrelación docente-alumno se aprecia que entre el nivel primaria y secundaria se ubicaron en la categoría "Buena" el 61.29\%, en la categoría "Regular" se ubicó el 33.87\% y en la categoría "Mala" solo un 4.84\%. De acuerdo a la información presentada se interpreta que los docentes tanto del nivel primaria y secundaria evidencian una buena interrelación con los alumnos puesto que se relacionan de forma asertiva y empática durante el desarrollo de las actividades dentro de la institución, así mismo el docente en conjunto con los alumnos buscan soluciones a los conflictos que surjan en función a los valores y los acuerdos de convivencia establecidos al inicio del año escolar.

En el nivel secundario, si bien el porcentaje mayor se ubicó en la categoría "buena" con un $54.14 \%$ se destaca que los docentes de este nivel demostraron que existen algunas debilidades para promover un ambiente de respeto y diálogo entre los que intervienen en el proceso de enseñanzaaprendizaje.

Acerca del Nivel de manejo de recursos que presentan los docentes se aprecia que entre el nivel primaria y secundaria el $58.06 \%$ se ubicó en la categoría "Adecuada" y el $41.94 \%$ se ubicó en la categoría "Inadecuada". Los recursos representan el conjunto de elementos y bienes disponibles del aula para desarrollar actividades dentro de la institución. El manejo de esos recursos, por parte del docente, es la manera óptima en la que distribuye los materiales y ambientes para dar paso a las sesiones de aprendizaje planificadas. Bajo esta premisa los resultados del nivel primaria evidencian que solo un $55.56 \%$ considera que el manejo de los recursos es adecuado debido a que existen algunas debilidades en la elaboración de material por parte del docente puesto que no están del todo articulados o en función de las necesidades de aprendizaje, sino más bien de los contenidos. De igual forma el nivel secundaria evidencia que un $60.00 \%$ maneja los recursos de la institución adecuadamente, presentando dificultades para manejar con pertinencia los recursos educativos.

Debido a lo mencionado es que el manejo de recursos puede presentar mejoras a través de acciones que fortalezcan la elaboración de material educativo en función de una educación de calidad para los estudiantes.

En cuanto al Nivel de administración de la evaluación que presentan los docentes se observa que entre el nivel primaria y secundaria el $62.90 \%$ se ubicó en la categoría "Regular", en la categoría "Buena" se ubicó el $25.81 \%$. En función a la información presentada se interpreta que la administración de la evaluación que realizan los docentes tanto del nivel primaria y secundaria es regular con un $51.85 \%$ y $71.43 \%$ respectivamente debido a que en el nivel primaria las unidades de estudio manifiestan que tienen dificultades para realizar evaluaciones personalizadas, es decir, evaluar de acuerdo a los estilos y ritmos de aprendizaje de cada uno de los estudiantes y en el nivel secundaria las deficiencias son debido a que no siempre utiliza los resultados de las evaluaciones para 
retroalimentar a los estudiantes. Sin embargo, los docentes en ambos niveles educativos manifestaron que emplean evaluaciones formativas debido a que el enfoque de la educación requiere del desarrollo de las capacidades y medir el logro alcanzado por los estudiantes.

Tabla 4

Nivel de desempeño docente en la I.E. Manuel A Odria de Tacna en el año 2018.

\begin{tabular}{lcccccc}
\hline & \multicolumn{2}{c}{ Primaria } & \multicolumn{2}{c}{ Secundaria } & \multicolumn{2}{c}{ Total } \\
\hline Categorías & $\mathrm{f}$ & $\%$ & $\mathrm{f}$ & $\%$ & $\mathrm{f}$ & $\%$ \\
\hline Desempeño docente adecuado & 17 & 62.96 & 15 & 42.86 & 32 & 51.61 \\
Desempeño docente no adecuado & 10 & 37.04 & 20 & 57.14 & 30 & 48.39 \\
Total & 27 & 100.00 & 35 & 100.00 & 62 & 100.00 \\
\hline
\end{tabular}

Fuente: cuestionario aplicado a docentes

La tabla 4 presenta información sobre el Nivel de desempeño docente, así se aprecia que un $51.61 \%$ se ubicaron en la categoría "Desempeño docente adecuado" y en la categoría "Desempeño docente no adecuado" un 48.39\%. El nivel de desempeño de los docentes que laboran en la I.E. Manuel A. Odría de Tacna en el año 2018 y que participaron en los Grupos de Interaprendizaje, evidencia, en términos generales, una mejora. Sin embargo, en el nivel secundaria las mejoras en el desempeño docente solo alcanzaron un $42.86 \%$ siendo superado por el porcentaje que se ubicó en la categoría "Desempeño docente no adecuado" un 57.14\% por lo tanto la aplicación de los GIA en el nivel secundaria refleja debilidades considerables en el manejo de recursos y la administración de la evaluación de los estudiantes.

Tabla 5

Nivel de mejora del desempeño docente en la l.E Manuel A. Odría

\begin{tabular}{lcccccc}
\hline & \multicolumn{2}{c}{ Primaria } & \multicolumn{2}{c}{ Secundaria } & \multicolumn{2}{c}{ Total } \\
\hline Categorías & $\mathrm{f}$ & $\%$ & $\mathrm{f}$ & $\%$ & $\mathrm{f}$ & $\%$ \\
\hline Alto & 0 & 0.00 & 2 & 13.33 & 2 & 6.25 \\
Moderado & 5 & 29.41 & 4 & 26.67 & 9 & 28.13 \\
Leve & 12 & 70.59 & 9 & 60.00 & 21 & 65.62 \\
Total & 17 & 100.00 & 15 & 100.00 & 32 & 100.00 \\
\hline
\end{tabular}

Fuente: Cuestionario aplicado a docentes

La tabla 5 presenta información sobre el Nivel de mejora del desempeño docente en la I.E. Manuel A. Odría en el año 2018, en ella se aprecia que el $65.62 \%$ se ubicó en la categoría "Leve", el $28.63 \%$ en la categoría "Moderado" y el $6.25 \%$ se ubicó en la categoría "Alto". De acuerdo a la información presentada se interpreta que en el nivel primaria ningún docente presentó un alto nivel de mejora en su desempeño docente, caso contrario un $70.59 \%$ lo logró solo levemente, del mismo modo en el nivel secundaria un $60.00 \%$ de los docentes se ubicó en la categoría "Leve", por lo que entre el nivel primaria y secundaria los docentes, si evidenciaron la presencia de un nivel de mejora en su desempeño docente, leve.

Para comprobar la relación existente entre las variables aplicación de Grupos de Interaprendizaje y desempeño docente, se trabajó con el coeficiente de correlación Rho de Spearman. La aplicación de la estrategia "Grupos de Interaprendizaje" (GIA) en la Institución Educativa Manuel A. Odría es óptima en un porcentaje levemente significativo (51.61\%) y que su desempeño, también presenta un porcentaje similar (51.61\%) indicando que hay mejora; sin embargo, ante estos resultados y lo obtenido a través del coeficiente de correlación de Rho de Spearman permite señalar que no existe 
una relación significativa. La relación que se observa entre las dos variables puede ser calificada de leve o baja.

De acuerdo a lo expuesto en los párrafos anteriores, se puede considerar que el impacto de la aplicación de los GIA, sólo es moderado ya que no se establece una relación entre las variables del estudio. La conclusión a la que se ha llegado, se ve fortalecida con la investigación realizada por Rodríguez (2015) en la que concluye que los docentes que participan en los GIA se vieron influenciados positivamente habiéndose reforzado así su desempeño docente. De igual forma, la intención de investigar sobre el desempeño docente, se refuerza con los resultados alcanzados por la investigación realizada por Mérida (2018) donde se destaca la importancia de la evaluación de esta característica en el profesor. Finalmente, en nuestra realidad, la presente investigación llega a establecer que la aplicación del GIA en la Institución Educativa Manuel A. Odría ha generado un impacto moderado en el desempeño docente de los profesores que participaron en él.

\section{CONCLUSIONES}

Se ha logrado determinar que el grado de aplicación de la estrategia "Grupos de Interaprendizaje" (GIA) en la institución educativa ha sido óptimo en un 51.61\%. Sin embargo, en esta categoría, sólo la aplicación de los GIA, logra sobrepasar el $50 \%$ en su planificación (51.61\%) y se encuentran por debajo de este porcentaje indicadores como: distribución del tiempo empleado (32.26\%), Organización (46.77 $\%)$, Recursos empleados $33.87 \%$ y nivel de control (40.32\%). Se ha podido establecer que al término de la aplicación de la estrategia "Grupos de Interaprendizaje", en la institución, el nivel de desempeño docente que presentan, se ubica en un nivel de mejora leve (65.62\%) seguido del nivel moderado $(28.13 \%)$ y en el nivel alto (6.25\%). Este nivel de mejora se traduce en un $51.61 \%$ de docentes que han logrado ubicarse en esta categoría. Sin embargo, su desempeño presenta debilidades en su planificación (54.84\%), en la ejecución del proceso enseñanza aprendizaje (33.87\%), en la interrelación docente alumno (33.87\%), en el manejo de recursos didácticos (41.94\%) en la administración de la evaluación (62.90\%), que se ubican en la categoría regular o inadecuado. El resultado encontrado lleva a concluir que los docentes mejoran su desempeñó, aunque levemente con su participación en el GIA.

En la relación entre el grado de aplicación de la estrategia "Grupos de Interaprendizaje" y el desempeño docente, se establece que no hay una relación significativa entre las variables del estudio. Los resultados encontrados permiten concluir que el impacto de la aplicación de la estrategia "Grupos de Interaprendizaje" (GIA) en el desempeño en la institución educativa es moderado. Si bien es cierto existe una mejora en el desempeño con una aplicación de la estrategia GIA, pero es débil en cuanto sólo el $51.61 \%$ consideran que la aplicación ha sido positiva, se encuentra un $48.39 \%$ piensan que no lo ha sido. Sería conveniente que los responsables de la aplicación de los GIA tomen medidas para superar los factores la distribución del tiempo evitando la saturación que causa insatisfacción en los docentes, la organización de las actividades, el empleo de recursos de trabajo para el desarrollo de las actividades que se proponga en los GIA y el nivel de control de los aprendizajes para asegurar el logro de las competencias propuestas para el desempeño docente. (Murray et al., 2021)

\section{REFERENCIAS BIBLIOGRÁFICAS}

Chávez, M. (2017). Desempeño docente según estudiantes del VII ciclo de instituciones educativas técnicas del distrito de Pangoa - Satipo. Huancayo - Perú: UNCP.

Congreso. (2014). Ley 30220 Ley Universitaria. Lima: El Peruano.

Díaz, F., \& Hernández, G. (2004). Estrategias Docentes para un aprendizaje significativo. Una interpretación constructivista (2 ed.). México: McGraw - Hill.

Espinoza, J., Vilca, C., \& Pariona, J. (2014). El Desempeño docente y el rendimiento académico en el curso de aritmética:conjuntos, Lógica proposicional del cuarto grado de secundaria de la

Ivancevich, J. (2005). Administración de recursos humanos. México: Mc Graw-Hill. 
Kozak, D., \& Novello, J. (2003). Proyecto Aulas en Red/ Escuelas con Intensificación en Tecnologías de la Información y la Comunicación. Recuperado el 4 de enero de 2019, de https://www.buenosaires.gob.ar/areas/educacion/niveles/primaria/programas/aulasenred/ pdf/dossier_circulo_aprendizaje.pdf

Mérida, V. (2018). Evaluación del desempeño docente en el sector público. Quetzaltenango: Universidad Rafael Landívar.

Ministerio de Educación de Colombia. (2017). Círculos de calidad: Redes de aprendizaje. Guias de Trabajo para las Instituciones Educativas. Recuperado el 15 de enero de 2018, de https://educacion.gob.ec/wp-content/uploads/downloads/2017/04/CIRCULOS-DECALIDAD.pdf

Ministerio de Educación del Perú. (2012). Marco del buen desempeño docente. Lima: Minedu.

Ministerio de Educación del Perú. (2015). Manual para los grupos de interaprendizaje -GIA. Lima: Minedu. Recuperado el 20 de diciembre de 2018, de http://redined.mecd.gob.es/xmlui/bitstream/handle/11162/61620/00820082006001.pdf?se quence $=1 \&$ isAllowed $=y$

Ministerio de Educación del Perú. (2017). Asesoría a la gestión escolar y CIAG-Orientaciones, protocolo $e$ instrumentos. Lima. Recuperado el 8 de diciembre de 2018, de http://www.minedu.gob.pe/curso-diplomadoysegundaespecialidad-directivos/pdf/mod4/gciag-participante-f3.pdf

Ministerio de Educación del Perú. (2018). Orientaciones y protocolos para el desarrollo de estrategias Formativas del programa de formación en servicio dirigido a docentes de II.EE del nivel primaria con acompañamiento pedagógico. Lima.

Montenegro, A., \& Abdón, I. (2003). Evaluación del desempeño docente: fundamentos, modelos e instrumentos. Bogotá: Cooperativa Editorial Magisterio.

Rodriguez, A. (2010). Diseño de Guias de Interaprendizaje para el desarrollo de seminarios-talleres de Artes Visuales. Quito: Autor. Recuperado el 10 de diciembre de 2018, de file:///C:/Users/dayana/Downloads/T-PUCE-3532.pdf

Rodríguez, L. (2015). Acompañamiento pedagógico y grupos de Interaprendizaje en el desempeño docente - Ugel 03-Lima, 2015. Lima: autor. Recuperado el 14 de diceimbre de 2018, de http://repositorio.ucv.edu.pe/handle/UCV/4531

Zabalza M., A. (2007). Guía para el Desarrollo de Competencias Docentes. México: Trillas.

Zepeda, S. (2012). Professional development. What Works (Segunda ed.). Nueva York: Routledge. 